\title{
Fractal growth in impurity-controlled solidification in lipid monolayers
}

\author{
Fogedby, Hans C.; Sørensen, Erik Schwartz; Mouritsen, Ole G.
}

Published in:

Journal of Chemical Physics

Link to article, DOI:

$10.1063 / 1.453406$

Publication date:

1987

Document Version

Publisher's PDF, also known as Version of record

Link back to DTU Orbit

Citation (APA):

Fogedby, H. C., Sørensen, E. S., \& Mouritsen, O. G. (1987). Fractal growth in impurity-controlled solidification in lipid monolayers. Journal of Chemical Physics, 87(11), 6706-6709. https://doi.org/10.1063/1.453406

\section{General rights}

Copyright and moral rights for the publications made accessible in the public portal are retained by the authors and/or other copyright owners and it is a condition of accessing publications that users recognise and abide by the legal requirements associated with these rights.

- Users may download and print one copy of any publication from the public portal for the purpose of private study or research.

- You may not further distribute the material or use it for any profit-making activity or commercial gain

- You may freely distribute the URL identifying the publication in the public portal

If you believe that this document breaches copyright please contact us providing details, and we will remove access to the work immediately and investigate your claim. 


\title{
Fractal growth in impurity-controlled solidification in lipid monolayers
}

Hans C. Fogedby and Erik Schwartz Sørensen

Institute of Physics, University of Aarhus, DK-8000 Aarhus C, Denmark

Ole G. Mouritsen

Department of Structural Properties of Materials, The Technical University of Denmark, Building 307, DK-2800 Lyngby, Denmark

(Received 8 July 1987; accepted 28 August 1987)

\begin{abstract}
A simple two-dimensional microscopic model is proposed to describe solidifcation processes in systems with impurities which are miscible only in the fluid phase. Computer simulation of the model shows that the resulting solids are fractal over a wide range of impurity concentrations and impurity diffusional constants. A fractal-forming mechanism is suggested for impuritycontrolled solidification which is consistent with recent experimental observations of fractal growth of solid phospholipid domains in monolayers.
\end{abstract}

\section{INTRODUCTION}

Solidification in fluid systems far from equilibrium is known to involve formation and growth of patterns with different morphologies. The nature of the morphology will depend on the mechanism by which the solidifying material is transported to the interface, the tension of the fluid-solid interface, as well as on the diffusion of latent heat away from the solidification front. Examples include (a) compact crystalline domains resulting from thermal quenches of a pure liquid below its melting point, and (b) dendritic and tipsplitting structures formed from a nucleation site in a supercooled fluid or liquid solution. ${ }^{1}$ In the first example, the crystalline domains grow compact because the solidifying process is controlled by a stable interface. In the second example, the structures become tenuous, or fractal, because the growth is controlled by diffusion and interface instabilities. ${ }^{1,2}$ A variety of other growth processes lead to similar morphologies, e.g., aggregation, ${ }^{3-5}$ viscous fingering, ${ }^{6-8}$ electrochemical deposition, ${ }^{9,10}$ and amorphous annealing. ${ }^{8}$ There is currently a strong interest in unravelling possible common principles underlying these apparently unrelated phenomena and, in particular, in determining the parameters which control the distinct morphologies and the crossover between one type of morphology and another.

In this paper we shall discuss solidification processes in fluid systems where the growth-limiting factor is diffusion of a second component, such as an impurity or a solvent which is miscible in the fluid phase only. We shall work in terms of a "minimal model" which seeks to isolate the bare effects of chemical diffusion, thus neglecting the interface tension and the heat of solidification. In this case, the solidification may be viewed as a particular aggregation process. We have a special class of systems in mind for our modelling: lipid monolayers at an air/water interface. As a function of lateral pressure, two-dimensional lipid monolayers pass through a series of phase transitions, one of which takes the monolayer from a liquid- to a solid-condensed state. ${ }^{11}$ In the presence of a dye impurity, which is miscible only in the fluid phase, the nucleation and growth of solid domains with distinct fractal morphologies has recently been observed by fluorescence microscopy, subsequent to an upward jump in lateral pressure. ${ }^{12,13}$ Since fractal patterns are not expected in the absence of the dye, ${ }^{13}$ and since the heat of solidification is im- mediately transferred to the water subphase, ${ }^{14}$ there are strong indications that it is the diffusive characteristics of the dye impurity which are responsible for the morphology of the solid domains. It is the purpose of the present paper, by means of a simple model, to demonstrate that this may indeed be the case.

\section{THE MODEL}

The model is formulated as a microscopic aggregation process on a lattice with particles and it is related to the multiparticle diffusive aggregation model (MPDA) discussed by Meakin and Deutch ${ }^{15}$ and by Voss. ${ }^{16}$ It differs from previous theoretical approaches to aggregation processes in three principle ways: (i) it accounts microscopically for a second component (the voids in MPDA ${ }^{16}$ ), (ii) the aggregating particles are all introduced simultaneously and not one by one as in standard models of diffusion-limited aggregation (DLA), ${ }^{5}$ and (iii) particles at the surface of the aggregate are added with a probability which in the general case is less than unity.

The growth process in the model is assumed to be governed by two mechanisms: (i) irreversible solidification of fluid particles at the solid-fluid interface, and (ii) chemical diffusion of impurities (or solvent molecules) in the fluid phase only. Hence, the growth model is characterized by two intrinsic timescales, that of fluid-solid conversion $\left(\tau_{S}\right)$ and that of impurity diffusion $\left(\tau_{D}\right)$. The two important model parameters are, therefore, the timescale ratio $\tau_{S} / \tau_{D}$ and the impurity concentration $C$. The nonequilibrium circumstances for the growth are similar to those of constitutional supercooling in metallurgy. ${ }^{17}$ The microscopic realization of the model is on an $L \times L$ square lattice, where each site is occupied by either a solid particle, a fluid particle, or an impurity ( formally treated as a site vacancy). The initial configuration of the system consists of a random mixture of fluid particles and impurities corresponding to the chosen concentration. A compact solid seed is placed at the origin of the lattice. The growth dynamics is then implemented as follows: Fluid particles, which are nearest neighbors to solid particles in the current solid aggregate (or seed), are solidified and attached irreversibly (without release of latent heat) to the aggregate on a timescale $\tau_{s}$. Nearest-neighbor pairs of fluid and impurity particles are made to interchange 
(diffuse) on the time scale $\tau_{D}$. The growth model may be considered a generalized Eden model ${ }^{18}$ in which all particles at the current aggregate surface have the same probability of sticking, but the growth is limited by the impurities which may momentarily cover surface sites to an extent which depends on the local impurity concentration near the solidification front. It is these impurities and their diffusional characteristics which cause the interfacial instability of the present growth model and are responsible for the morphology of the resulting aggregates.

\section{RESULTS}

We have grown aggregates to average linear sizes ranging from 100 to 500 lattice units enclosed in a mask of variable size in order to eliminate effects of the boundaries. Different seed sizes and shapes have been used, and ensemble average properties are obtained by averaging over the results derived from several different sequences of the random numbers used to simulate the stochastic nature of the solidification and the diffusional motions. In Fig. 1 are shown typical solid aggregates grown to a size of about 150 lattice units at impurity concentration $C=0.7$ for a series of time scale ratios. For very fast diffusion, the aggregates exhibit moderate tipsplitting with a characteristic finger thickness; in the limit of slow diffusion, the tipsplitting is more pronounced but the aggregates grow with a lower density. For intermediate diffusion rates, the aggregates are tenuous and in appearance similar to those observed in standard DLA and MPDA. ${ }^{16}$ The tenuity of the aggregates and their possible scale invariance are conveniently analyzed quantitatively in terms of a fractal (or Hausdorff) dimension $D$ defined by ${ }^{19}$

$$
N(R) \sim R^{D},
$$

where $N(R)$ is the number of particles in a region of the aggregate of linear extension $R$. In Fig. 2 we show that the aggregates do form scale-invariant patterns over a wide range of distances. There is, of course, low- $R$ and high- $R$ cutoffs and crossover regions due to interference with the smallest dimension of the lattice and the finite size of the aggregate, respectively. The full variation of $D$ over a wide range of timescale ratios is shown in Fig. 3. $D$ is seen to vary in a seemingly continuous manner.

Preliminary results obtained for other impurity concentrations ranging from $C=0.5$ to $C=0.9$ are found to be qualitatively similar to those presented in Figs. 1-3 for

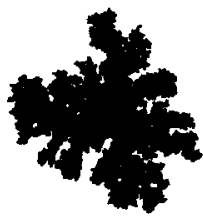

a

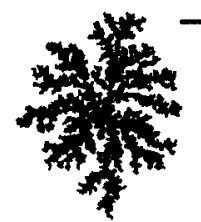

b

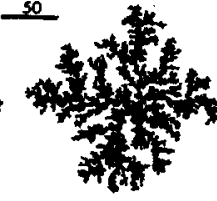

C

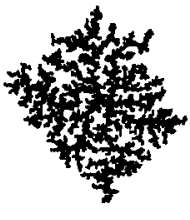

c
FIG. 1. Typical aggregates grown to a size of about 150 lattice units for impurity concentration $C=0.7$ at different timescale ratios, $\tau_{s} / \tau_{D}=10$ (a), 1.4 (b), 0.8 (c), and 0.1 (d). The aggregates consist, respectively, of about $13000,7000,6200$, and 5500 particles. The solid bar indicates 50 lattice units.

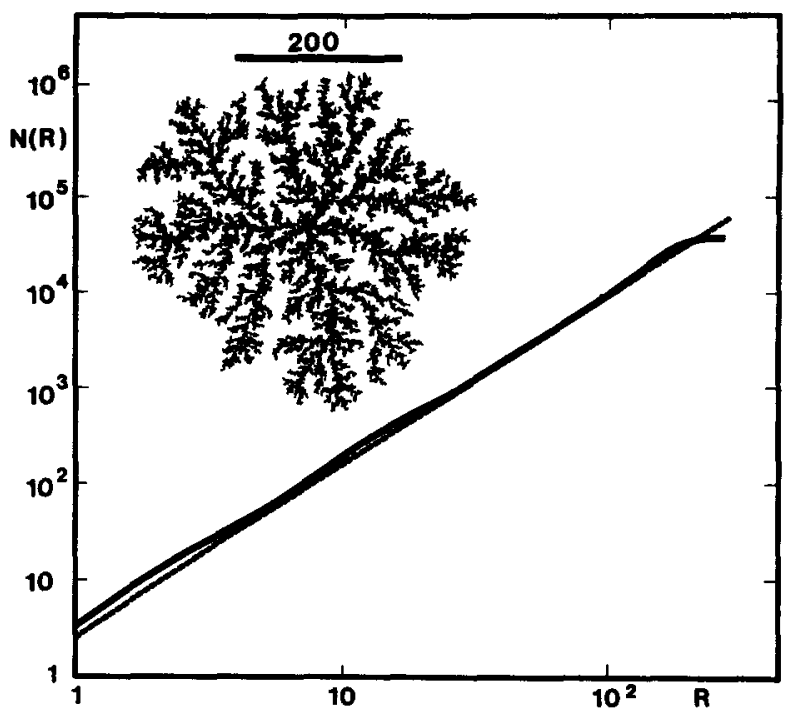

FIG. 2. Double-logarithmic plot of the particle content, $N(R)$, vs linear extension, $R$, of a very large aggregate consisting of about 35000 particles grown at $\tau_{S} / \tau_{D}=1$ and $C=0.7$. This aggregate is shown on a scale indicated by the solid bar which corresponds to 200 lattice units.

$C=0.7$. It is observed that when $C$ is increased within this interval, for a fixed value of $\tau_{S} / \tau_{D}$, the effective fractal dimension decreases corresponding to more tenuous aggregates. This behavior is in agreement with Voss ${ }^{16}$ who finds a crossover from fractal to uniform aggregates at a correlation length which increases with the vacancy concentration. For large $C$, the fractal dimension $D$ approaches the DLA value of 1.7, corresponding to the decrease of the fluid-fluid particle correlations. This is also evident from the plot in Fig. 2 which shows that the effective fractal dimension is smaller at the intermediate stages of the growth where the local impurity concentration is larger. In the long-time limit, the aggregate grows fjords which trap the impurities and lead to a steady state corresponding to a lower fractal dimension.

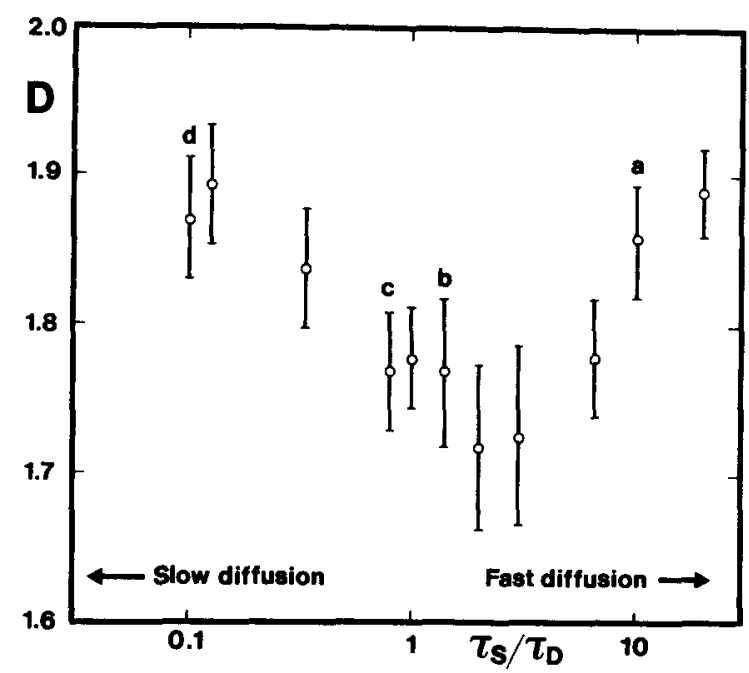

FIG. 3. Semilogarithmic plot of the fractal dimension $D$, Eq. (1), vs time scale ratio $\tau_{S} / \tau_{D}$ for solidification and impurity diffusion with impurity concentration $\boldsymbol{C}=0.7$. The plotted points are mean values obtained by averaging over 10 to 15 samples. On the plot are also shown the values of $D$ for the aggregates shown in Fig. 1. 
More calculations than presented here will be needed to assess quantitatively the influence of the value of $C$ on the growth characteristics in the various time regimes and whether some universal principle might be operative. It may seem that the values of the "impurity" concentration studied here are unphysically high. Two things should be kept in mind, however. (1) The second component of our binary system could be interpreted as a solvent. (2) The actual chemical activity of a solid-immicible impurity is likely to be much larger than the molar concentration; e.g., thinking of the impurity as a crystal breaker it is likely to "dissolve" the solid more effectively than just by excluded-volume effects.

The actual fractal-forming mechanism of the present model of solidification may be revealed by studying the spatial distribution of impurities as the growth process evolves. It turns out that this distribution is not spatially uniform and that the active growth zone is characterized by an elevated level of impurities which are being pushed away from the solidification front. Similarly, highly concentrated regions of impurities are being trapped between the arms of the fractal structure in the interior of the aggregate. This behavior is strongly reflected in the shape of the circularly averaged radial distribution function of impurities (measured from the center of the original seed) as pictured in Fig. 4. This function has a maximum near the active zone of growth. ${ }^{20}$ At any given time, it rises from the center of the aggregate and levels off to the uniform background concentration outside the aggregate. The maximum moves out as time lapses, Fig. 4, indicating that a halo of a locally high impurity concentration is driven by the solidification front. This halo acts as a fractal former in the solidification process and therefore plays a role analogous to that of the screening effect in DLA. ${ }^{5}$ Similarly, it is analogous to the depletion zone in MPDA. ${ }^{15,16}$

\section{DISCUSSION}

We now turn to a discussion of our results, firstly in relation to other recent model studies of fractal and dendritic growth, with particular emphasis on the role of anisotropy, and secondly in relation to experimental investigations of

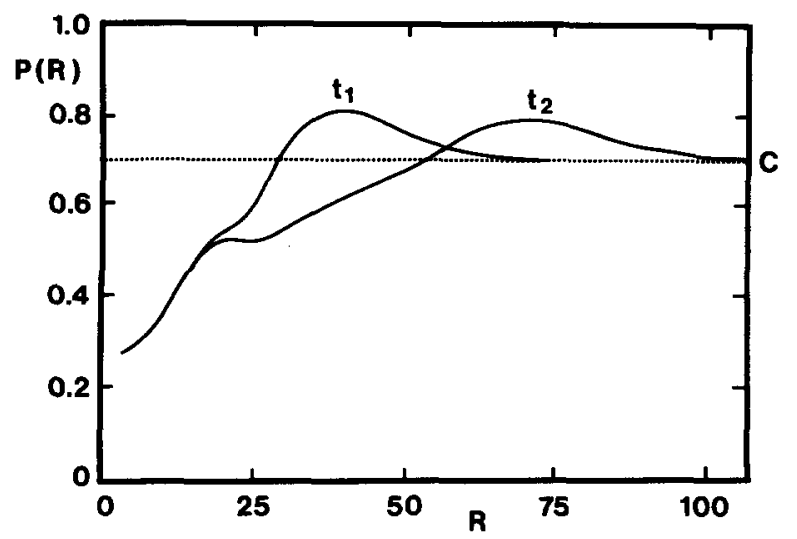

FIG. 4. The circularly averaged radial distribution function of impurities $P(R)$ as a function of time $t$ averaged over several samples. $P(R)$ and $t$ are in arbitrary units. The results are obtained for $C=0.7$ and $\tau_{S} / \tau_{D}=2$. For two consecutive times, $t_{1}<t_{2}$, the aggregate contains 1500 and 5000 particles, respectively. fractal solidification in lipid monolayers. One of the key issues in recent theoretical ${ }^{8,20-26}$ (and experimental ${ }^{6-8}$ ) studies of diffusion-limited growth has been to unravel the effects on the fractal dimension of (i) noise-induced instabilities in the solid-fluid interface, and (ii) spatial anisotropy in the diffusion process (possibly reflecting the molecular anisotropy of the aggregating particles). For DLA processes, it turns out that the level of noise (the noise being induced by the stochastic nature of the diffusing particles) is irrevelant for the value of $D$, except in the zero-noise limit. ${ }^{23}$ Anisotropy changes the isotropic DLA dimension, $D \simeq 1.7$, towards $D \simeq 1.5$ (dendritic growth), ${ }^{23-25}$ and possibly all the way to $D=1$ (needle growth) for extreme anisotropy. 22, 24 Whether these changes are gradual, or they conceal a few discrete universality classes each with their value of $D$, remains at present unclear.

For the present model we find, as shown in Fig. 3, that the fractal dimension $D$ depends on the timescale ratio $\tau_{s} /$ $\tau_{D}$ which seems to play the same role as the anisotropy parameter introduced by Jullien et al..$^{22}$ In the limit of a slow diffusion, corresponding to a low noise level, the solid phase penetrates the nearly static impurity matrix and attains a structure corresponding to the fluid-impurity composition prior to the growth process. This explains the increase in $D$ towards 2 (compact growth). Furthermore, from Fig. 1 we note that similar to large DLA aggregates, ${ }^{3}$ the present aggregates are also unstable to the underlying square lattice structure (indicating a change in universality class for large aggregates). At intermediate diffusion rates, $D$ exhibits a minimum value at about the DLA value 1.7 and the appearance of the aggregates is quite DLA-like. In the limit of fast diffusion we seem to approach the Eden limit. The aggregates become more compact exhibiting less finger splitting, a characteristic finger thickening, and $D$ approaches 2 .

A unique class of two-dimensional systems exists which might be a physical realization of the model proposed in this paper. These systems are lipid monolayers ${ }^{11}$ spread on an air/water interface in the presence of a lateral pressure. It was recently found ${ }^{12,13}$ that, within the experimental conditions used for a monolayer of dimyristoylphosphatidyl ethanolamine, a window in space and time exists for observing fractal growth of solid lipid domains characterized by $D \simeq 1.5$. This value need not be inconsistent with the results of the present model considering (i) the early-time regime in Fig. 2 is described by a lower effective exponent, and (ii) the long-time, possibly fractal, regime in the experimental system is wiped out due to approach to equilibrium. ${ }^{12,13}$ More importantly, the experimental study also found increased levels of fluorescent impurity molecules between the arms of the fractal aggregate and in the active growth zone, ${ }^{12,13}$ suggesting the same fractal-forming mechanism as in the model proposed here. It should be pointed out that our model study predicts that the experimentally observed value of $D$ may , well depend on details of the system which, for the present experimental system would be size of dye molecule $\left(\tau_{D}\right)$ and the type of lipid molecule $\left(\tau_{s}\right)$.

Ben-Jacob et al. ${ }^{8}$ recently argued that DLA-like morphologies may be viewed as the vanishing-effective-surfacetension limit of a dense branching morphology which is not 
fractal. The dense branching morphology is characterized by a circular envelope with leading branch tips controlled by a fastest growing mode. These authors also surmised that the solid patterns formed in lipid monolayers have the dense branching morphology and are thus not fractal. Further analysis of the experiments is required to settle this question.

Finally, it should be noted that the model for impuritycontrolled solidification put forward in this paper is per construction only describing far-from-equilibrium situations and is thus unable to account for the late stages of the solidification process where compact solid domains eventually will be formed (as is also invariably observed in the monolayer experiments ${ }^{13}$ ). However, the model could be extended to account for this phenomenon too by allowing the solidified particles to melt and dissolve again as governed by an appropriate finite-temperature activation process.

\section{ACKNOWLEDGMENTS}

This work was supported by the Danish Natural Science Council under Grants No. J.nr. 5.21.88.72 and J.nr. 115593. We wish to thank Helmuth Möhwald for stimulating discussions on his experimental observations of fractal growth of phospholipid domains in monomolecular layers and Paul Meakin for pointing out to us the relation between our model and those of Refs. 15 and 16.

${ }^{1}$ J. S. Langer, Rev. Mod. Phys. 52, 1 ( 1980); Phys.Rev. A 33, 435 (1986). ${ }^{2}$ On Growth and Form, edited by H. E. Stanley and N. Ostrowsky (Martinus Nijhoff, Boston, 1986).

${ }^{3}$ T. A. Witten and M.E, Cates, Science 232, 1607 ( 1986).

${ }^{4}$ L. M. Sander, Nature 322, 789 (1986).
${ }^{5}$ T. A. Witten and L. M. Sander, Phys. Rev. B 27, 5686 ( 1983 ).

${ }^{6} \mathrm{E}$. Ben-Jacob, R. Godney, N. D. Goldenfeld, J. Koplik, H. Levine, T. Mueller, and L. M. Sander, Phys. Rev. Lett. 55, 1315 (1985).

${ }^{7}$ J. Nittmann, C. Daccord, and H. E. Stanley, Nature 314, 141 (1985).

${ }^{8}$ E. Ben-Jacob, C. Deutscher, P. Garik, N. G. Goldenfeld, and Y. Lareah, Phys. Rev. Lett. 57, 1903 (1986).

${ }^{9}$ Y. Sawada, A. Dougherty, and J. P. Gollub, Phys. Rev. Lett. 56, 1260 (1986).

${ }^{10}$ D. Grier, E. Ben-Jacob, R. Clarke, and L. M. Sander, Phys. Rev. Lett. 56, 1264 (1986)

${ }^{1}$ O. Albrecht, H. Gruler, and E. Sackmann, J. Phys. (Paris) 39, 301 (1978).

${ }^{12}$ A. Miller, W. Knoll, and H. Möhwald, Phys. Rev. Lett. 56, 2633 (1986).

${ }^{13}$ A. Miller and H. Möhwald, J. Chem. Phys. 86, 4258 (1987).

${ }^{14}$ The solidification of the lipid monolayer takes place predominantly in terms of the acyl-chain conformational degrees of freedom. The characteristic time of conformational changes from a disordered chain to the conformationally ordered chain characteristic of the solid is of the order of $10^{-6} \mathrm{~s}$. The thermal link to the water subphase via the polar head groups is, on the other hand, established within the nanosecond regime [see, e.g., A. Genz and J. F. Holzwarth, Eur. Biophys. J. 13, 323 (1986) ]. Thus the water subphase acts as a very effective sink for the latent heat released during solidification processes in the lipid monolayer.

${ }^{15}$ P. Meakin and J. M. Deutch, J. Chem. Phys. 80, 2115 (1983).

${ }^{16}$ R. F. Voss, Phys. Rev. B 30, 334 (1983).

${ }^{17}$ W. A. Tiller, K. A. Jackson, J. W. Rutter, and B. Chalmers, Acta Metall. 1, 428 (1953).

${ }^{18} \mathrm{M}$. Eden, Proceedings of the Fourth Berkeley Symposium on Mathematics Statistics and Probability, edited by J. Neuman (University of California, Berkeley, 1961), Vol. 4, p. 233.

${ }^{19}$ B. B. Mandelbrot, The Fractal Geometry of Nature (Freeman, San Francisco, 1982).

${ }^{20} \mathrm{Z}$. Rácz and M. Plischke, Phys.Rev. A 31, 985 (1985).

${ }^{21} T$. Vicsek, Phys. Rev.Lett. 53, 2281 (1984).

${ }^{22}$ R. Jullien, M. Kolb, and R. Botet, J. Phys. (Paris) 45, 395 ( 1984).

${ }^{23}$ J. Nittmann and H. E. Stanley, Nature 321, 663 (1986).

${ }^{24}$ J. Kertész and T. Vicsek, J. Phys. A 19, L257 (1986).

${ }^{25}$ O.-Y. Zhong-can, Y. Gang, and H. Bai-lin, Phys. Rev. Lett. 57, 3203 (1986).

${ }^{26}$ D. A. Kessler and H. Levine, Phys. Rev. Lett. 57, 3069 ( 1986). 